\title{
Role of Transarterial Embolization in the Management of Hepatic Artery Pseudoaneurysm Postlaparoscopic Cholecystectomy
}

\author{
Rozil Jayesh Gandhi ${ }^{1, \odot ~ K u n a l ~ B h a r a t ~ G a l a ~}{ }^{2, \odot} \quad$ Aditi R Gandhi ${ }^{1}$ \\ ${ }^{1}$ Department of Radiodiagnosis, Shardaben Municipality Hospital, \\ Address for correspondence Aditi R. Gandhi, MD, Shardaben \\ Ahmedabad, Gujarat, India \\ 2Department of Radiodiagnosis, Tata Memorial Hospital, Parel, \\ Mumbai, India \\ Municipality Hospital, Ahmedabad, Gujarat 380018, India \\ (e-mail: aditi257@yahoo.co.in).
}

J Clin Interv Radiol ISVIR:2020;4:148-153

\begin{abstract}
Purpose To assess the safety and effectiveness of transarterial embolization (TAE) in the management of hepatic artery pseudoaneurysms following laparoscopic cholecystectomy (LC).

Materials and Methods This is a retrospective study consisting of 13 patients who had undergone TAE for management of pseudoaneurysms of the hepatic artery or its branches following LC. Patients presented with symptoms such as hypotension, hematemesis, melena or gradual anemia post LC. Abdominal CT revealed hepatic artery pseudoaneurysm in all patients. TAE was performed with either coils or N-butyl cyanoacrylate or both. We evaluated technical and clinical effectiveness and compli-

Keywords

- hepatic artery pseudoaneurysm

- laparoscopic cholecystectomy

- trans arterial embolization

- vascular injury

- Coils

- N-Butyl cyanoacrylate (NBCA) cations of TAE.

Results TAE was successful both technically and in stopping bleeding in all patients ( $100 \%$ technical and clinical success). No patient had rebleeding after TAE. Four patients required laparotomy for draining hemoperitoneum and clots. Endoscopic retrograde cholangiopancreatography (ERCP) for hemobilia or bile duct injury was done in eight patients. Following TAE, hepatic infarct was observed in three patients. Postembolization syndrome was seen in four patients and one patient died due to sepsis.

Conclusions TAE is effective for treatment of hepatic artery pseudoaneurysms after LC. Hepatic infarcts and postembolization syndrome are the most common complications and can be managed conservatively.
\end{abstract}

\section{Introduction}

Laparoscopic cholecystectomy (LC) is now the gold standard for the treatment of symptomatic gall bladder diseases because it is associated with less postoperative pain, early discharge, and decreased wound complications as compared with open cholecystectomy. LC is known for a different set of complications as compared with open cholecystectomy, and those complications are more complex with injury to bile duct and adjacent vascular structures. Other injuries include iatrogenic trauma to bowel during access to the peritoneal cavity and retroperitoneal vascular injury with an overall morbidity of $2.9 \%{ }^{1-4}$ Major vascular complications following LC are rare, with an incidence of $0.25 \%$. The vascular injuries include intraoperative bleeding following injury to the cystic artery,
DOI https://doi.org/ 10.1055/s-0040-1721533 ISSN 2457-0214.
(C2020. Indian Society of Vascular and Interventional Radiology. This is an open access article published by Thieme under the terms of the Creative Commons Attribution-NonDerivative-NonCommercial-License, permitting copying and reproduction so long as the original work is given appropriate credit. Contents may not be used for commercial purposes, or adapted, remixed, transformed or built upon. (https://creativecommons.org/licenses/by-nc-nd/4.0/).

Thieme Medical and Scientific Publishers Pvt. Ltd. A-12, 2nd Floor, Sector 2, Noida-201301 UP, India 
hepatic artery, the portal vein or postoperative pseudoaneurysms (PSAs) of the cystic or hepatic arteries. ${ }^{4-7}$ Patients with PSA may be relatively asymptomatic and may present with mild anemia, but many present emergently with hypotension due to hematemesis, hemobilia or melena. ${ }^{6,8,9}$ These complications can occur during both in the early and late postoperative periods. A contrast-enhanced CT confirms the findings. TAE is for treatment of bleeding and PSAs with less morbidity and mortality compared with open surgery. ${ }^{5,9-11}$ A few studies have evaluated the role of TAE for postoperative PSAs following LC. These are limited to case reports and short reports. In this study, we report our experience of TAE for treatment of PSAs following LC in 13 consecutive patients.

\section{Materials and Methods}

This is a retrospective study of 13 patients who had undergone TAE for management of right hepatic artery PSA following LC between July 2016 to August 2019. There were 3 (23.07\%) females and 10 (76.9\%) men with the mean age of patients being 52 years (range 25-65 years). The indications for LC were as follows: acute calculus cholecystitis in six, chronic calculus cholecystitis in three, acalculous cholecystitis in two, and symptomatic cholelithiasis in two. The main complaints of patients were hematemesis, melena, abdominal pain, and gradual anemia. Some patients were admitted for symptoms of sepsis and biliary leak in the postoperative period (-Table 1). Acute presentation was hypotension due to hemoperitoneum, which was seen in four patients (30.7\%), hematemesis in three patients (23.07\%), melena in two patients (15.38\%), and gradual anemia in four patients (30.7\%). These symptoms occurred between 9 and 30 days post LC. The average reduction of the hemoglobin was $2.2 \mathrm{mg} /$ $\mathrm{dl}$ (range $1-3.5 \mathrm{mg} / \mathrm{dl}$ ). The diagnosis of PSA was made on duplex ultrasound in one patient and confirmed on multiphasic abdominal CT scan in all patients. Nonenhanced CT showed hemoperitoneum in four patients (30.7\%) and fluid collection/hematoma in the gall bladder fossa in six (46.1\%). On the arterial phase of contrast enhanced CT, a PSA of right hepatic artery was seen in 12 (92.3\%) patients. One patient (7.6\%) had complete occlusion of the right hepatic artery. No hepatic infarct was seen. Upper gastrointestinal (GI) endoscopy was performed when hematemesis or melena was present to rule out the other causes such as gastric ulcer or disease from the stomach or duodenum and confirm hemobilia.

\section{Embolization Procedure}

A common femoral arterial access was obtained and a $5 \mathrm{~F}$ vascular sheath was placed. A Simmons 1 (Sim1) or Cobra 2 (C2) (Cook Medical) catheter was used to select the superior mesenteric and celiac arteries. A digital subtraction angiography (DSA) was performed, and images were obtained during the arterial and portal venous phases. The angiogram was evaluated for presence of pseudoaneurysm, contrast extravasation, spasm of the artery, and arterial stenosis or irregularity near the pseudoaneurysm. After confirming the diagnosis of a PSA,

Table 1 Data analysis for hepatic artery pseudoaneurysm

\begin{tabular}{|c|c|c|c|c|c|c|c|}
\hline S.No. & Age & Gender & Symptoms & $\begin{array}{l}\text { Additional } \\
\text { procedure }\end{array}$ & $\begin{array}{l}\text { Embolization } \\
\text { material }\end{array}$ & $\begin{array}{l}\text { TAE on } \\
\text { postoperative } \\
\text { day }\end{array}$ & Complications \\
\hline 1 & 57 & M & Hematemesis & ERCP & NBCA & 30 & \\
\hline 2 & 25 & $\mathrm{M}$ & Melena & ERCP & Coils & 15 & \\
\hline 3 & 45 & $\mathrm{~F}$ & Sepsis + anemia & ERCP & Coils + NBCA & 12 & $\begin{array}{l}\text { Postembolization } \\
\text { syndrome }\end{array}$ \\
\hline 4 & 60 & $\mathrm{M}$ & Sepsis + anemia & ERCP & Coils & 18 & $\begin{array}{l}\text { Postembolization } \\
\text { syndrome }\end{array}$ \\
\hline 5 & 48 & $\mathrm{M}$ & Hematemesis & ERCP & Coils + NBCA & 22 & Liver infarcts \\
\hline 6 & 53 & $\mathrm{M}$ & Hypotension & ERCP & Coils + NBCA & 14 & \\
\hline 7 & 49 & $\mathrm{M}$ & Hypotension & $\begin{array}{l}\text { Laparotomy for } \\
\text { clot evacuation }\end{array}$ & Coils & 15 & \\
\hline 8 & 65 & M & Sepsis + anemia & ERCP & NBCA & 16 & $\begin{array}{l}\text { Postembolization } \\
\text { syndrome }\end{array}$ \\
\hline 9 & 55 & $\mathrm{~F}$ & Hypotension & $\begin{array}{l}\text { Laparotomy for } \\
\text { clot evacuation }\end{array}$ & Coils + NBCA & 18 & \\
\hline 10 & 59 & M & Hypotension & $\begin{array}{l}\text { Laparotomy for } \\
\text { clot evacuation }\end{array}$ & NBCA & 9 & \\
\hline 11 & 56 & $\mathrm{M}$ & Melena & ERCP & Coils + NBCA & 10 & Liver infarcts \\
\hline 12 & 55 & $M$ & Hematemesis & ERCP & NBCA & 11 & Liver infarcts \\
\hline 13 & 52 & $\mathrm{~F}$ & Sepsis + anemia & $\begin{array}{l}\text { Laparotomy for } \\
\text { clot evacuation }\end{array}$ & Coils + NBCA & 14 & $\begin{array}{l}\text { Postembolization } \\
\text { syndrome }\end{array}$ \\
\hline
\end{tabular}

Abbreviations: NBCA, n-butyl cyanoacrylate; TA, transarterial embolization. 
2.7 F Progreat microcatheter (Terumo) was advanced up to or beyond the PSA. The artery was dilated with 50 to 100 micrograms of nitroglycerine (NTG) before crossing it with a microcatheter. Depending upon the anatomy, microcoils (Hilal or Nester, Cook Medical) or n-butyl cyanoacrylate (NBCA) or both were used to occlude the artery and/or the PSA ( - Table $\mathbf{1}$ ). In four patients (31\%), the microcatheter could not cross the neck of the PSA, and the PSA was embolized ( - Fig. 1) with NBCA diluted to 20 to $50 \%$ with lipiodol (Guerbet). In three patients (23\%) embolization of the artery across the neck of the PSA was performed with only microcoils ( - Fig. 2). A combination of coils and NBCA, as the sandwich technique, was used in six patients $(46.1 \%)$, where coils were placed distally beyond the PSA and then to the PSA, which was treated with NBCA ( - Fig. 3). Such a combination was used to prevent nontarget embolization of NBCA into the distal hepatic arterial branches and increase the effectiveness of NBCA.

Nine patients $(9 / 13,69 \%)$ underwent endoscopic retrograde cholangiopancreatography (ERCP) and biliary stent for a concomitant biliary duct injury. Four patients $(4 / 13,31 \%)$ underwent laparotomy for clot evacuation ( - Table $\mathbf{1})$.

\section{Study Endpoints}

Patients were followed-up 1 week after TAE. The blood count and liver function test were obtained after 48 hours and after 7 days post TAE. The safety of TAE was assessed based on the incidence of major and minor complications, according to Society of Interventional Radiology (SIR) clinical practice
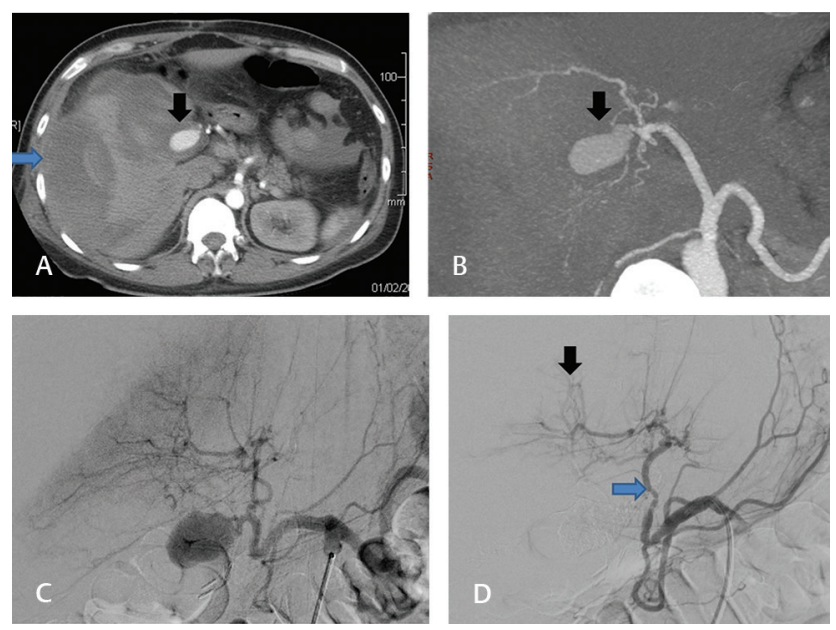

Fig. 1 (A) Computed tomography (CT) axial image showing large perihepatic hematomas (blue arrow) and oval contrast outpouching (black arrow) in the area of liver hilum suggestive of pseudoaneurysm. (B) CT maximum intensity projection (MIP) rotated axial image showing pseudoaneurysm (black arrow) arising from right hepatic artery. (C) Digital subtraction angiogram (DSA) of celiac artery with $5 \mathrm{~F}$ diagnostic catheter showing pseudoaneurysm (black arrow) arising from right hepatic artery. (D) Postembolization, common hepatic angiogram showing complete nonvisualization of right hepatic artery as well as pseudoaneurysm. Left hepatic artery (blue arrow) is normal and visualized well. N-butyl cyanoacrylate (NBCA) was used for embolization. Right hepatic arterial branches (black arrow) are visualized to be filling through segment IV collaterals of left hepatic artery.
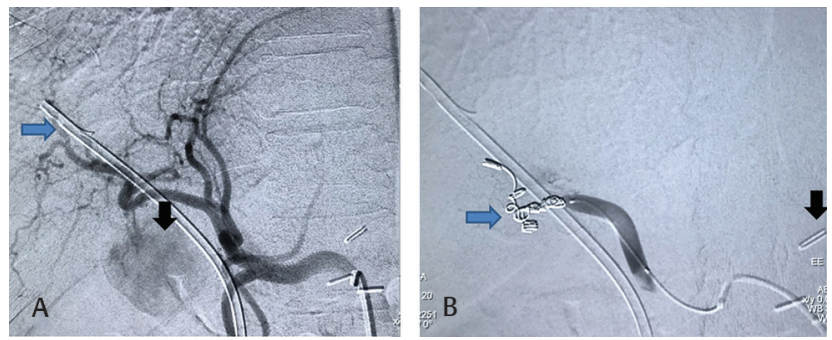

Fig. 2 (A) Common hepatic angiogram showing pseudoaneurysm (black arrow) filling from rent in right hepatic artery with jet of contrast seen. Plastic stent (blue arrow) inserted through Endoscopic retrograde cholangiopancreatography (ERCP) is seen. (B) Selective right hepatic angiogram from microcatheter after front to back coiling (blue arrow) of right hepatic artery segment at the level of pseudoaneurysm. Metallic surgical clips (black arrow) are seen far away from the pseudoaneurysm.

guidelines. ${ }^{12}$ The technical effectiveness of TAE was defined as the successful occlusion of the targeted vessel by the end of the embolization procedure. The clinical success was defined as relief of presenting symptoms. Rebleeding rates were assessed at 1 -week post TAE.

\section{Results}

DSA correlated with arterial phase CT scan in all patients. Bleeding site or pseudoaneurysm was found in all patients. Pseudoaneurysm was near the metallic clip in three patients (23.07\%) and away from the clip (>1 cm) in nine patients (69.2\%). No clip was found in one patient (7.6\%). A PSA was seen in 12 patients (92.3\%) which was concordant with the findings on CT scan. Active contrast extravasation, in addition to a PSA, was seen in five patients. The PSA was associated with narrowing and irregularity of the parent artery in all patients. In one patient, the right hepatic artery was occluded (abrupt cutoff) without a PSA. After negotiating with a microcatheter wire combination, the artery ruptured and contrast extravasation into the peritoneum. The artery was sealed with NBCA.

Microcoils were used in three patients (23.07\%), NBCA in four patients (30.7\%) ( - Fig. 4), and a combination of coils and NBCA was used in six patients (46.1\%). TAE had 100\% success rate in occluding the PSA and the parent artery. All patients stopped bleeding after TAE. There was no rebleeding at 7 days.

Postembolization syndrome (minor complication) was seen in four patients (30.7\%), who had presented with symptoms of abdominal pain, vomiting and fever postembolization ( - Table 1). These patients were managed conservatively. Hepatic infarcts (major complication) was seen in three patients (23.07\%). Liver infarcts were diagnosed by the rise in bilirubin levels and liver enzymes (more than three times of the baseline), with CT showing peripheral wedge-shaped hypodensity. ${ }^{13}$ They were all treated conservatively and responded well to medical management. One patient died postprocedure due to sepsis. 

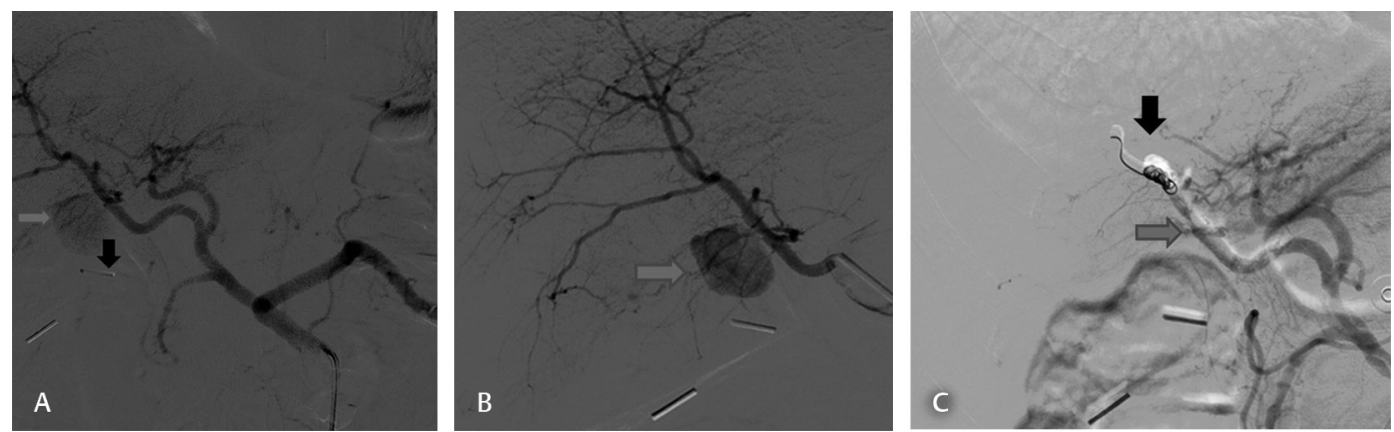

Fig. 3 (A) Celiac angiogram showing pseudoaneurysm (blue arrow) arising from right hepatic artery. Metallic clips (black arrow) are seen near the pseudoaneurysm. (B) Superselective cannulation of right hepatic artery and angiogram showing right hepatic artery (RHA) pseudoaneurysm (blue arrow). (C) Postembolization with coils (black arrow) and n-butyl cyanoacrylate (NBCA) (blue arrow) common hepatic angiogram showing no filling of pseudoaneurysm as well as right hepatic artery.

\section{Discussion}

The incidence of PSA arising from the hepatic or cystic artery after LC varies from $0.06 \%$ to $0.6 \%$ across the various studies. ${ }^{5,14}$ A $4.5 \%$ incidence of hepatic artery PSA is noted when there is an associated bile duct. ${ }^{6}$ The mechanisms involved in the formation of a PSA may be multifactorial and include excessive use of cauterization or mechanical injury during the dissection of Calot's triangle, particularly if there are any abnormal anatomical variations of the vasculature. ${ }^{4,7}$ The thermal injury can be due to either direct vessel injury by the cautery or indirectly via a metallic clip in contact with the artery. ${ }^{5}$ Bile acids dissolve the lipid membrane due to cytotoxic property, causing cell death in patients with bile leaks which can result in weakening of the arterial wall and PSA formation. ${ }^{6}$ Secondary infections due to bile leak also causes PSA.

Patients with hepatic artery pseudoaneurysm present with abdominal pain or discomfort, GI bleeding (hemobilia) either in the form of hematemesis or melena, alterations in the liver function tests (LFT) profile, or emergently with a hemoperitoneum. An abdominal mass with bruit may be detected on physical examination if the pseudoaneurysm is large. Rupture into the peritoneal cavity, portal vein, or pancreatic pseudocyst can also occur. ${ }^{15}$ However, the classical clinical triad described by Quinke in $1871^{16}$-right upper quadrant pain, jaundice, and hemobilia-has been reported in less than $40 \%$ of patients with LC-related hepatic artery pseudoaneurysm. ${ }^{14}$

The diagnosis of PSA is by history and physical examination, followed by upper GI endoscopy, since it will rule out the other causes of GI bleeding such as gastric ulcer or diseases of the stomach or duodenum. It is also helpful in identifying the blood clots or bleeding from major duodenal papilla. A multiphasic contrast CT is the best imaging test for detecting hepatic artery injuries following LC. According to Feng et al, $^{9}$ CT was highly useful and accurate in identifying the origin of PSA following LC. In this study, all patients had undergone CT scan, and a PSA was seen in 12 out of 13 patients which was confirmed on conventional angiography.
TAE is highly effective for managing hepatic artery pseudoaneurysms. ${ }^{9,15,17}$ In a literature review, Machado et al ${ }^{17}$ included 101 cases and 60 articles of PSA post LC. In this review, the most common artery involved was right hepatic artery $(87.1 \%)$ followed by cystic artery $(7.9 \%)$, both hepatic and cystic artery (4.0\%), and gastroduodenal artery (1.0\%). In our study, we encountered 12 patients with PSA arising from the right hepatic artery and one patient with right hepatic artery occlusion. Similar to prior studies, there was $100 \%$ success with TAE in our study. Unlike other studies, we used NBCA either alone or in combination with coils in 10 of 13 patients. We had one patient (25\%) who developed liver infarct post NBCA. The main disadvantage of NBCA is distal embolization. Hepatic artery spasm prevented selective catherization of the artery distal to the pseudoaneurysm in four patients, so embolization of the PSA was performed using NBCA from the proximal segment. In study by Chigot et al, ${ }^{18}$ percutaneous transhepatic embolization of PSA with NBCA was performed. This approach is useful when the artery supplying the PSA could not be catheterized endovascularly. In our study, six patients underwent embolization with coils followed by NBCA. The reasons for using coils with NBCA were that coils would prevent nontarget deposition of NBCA in the peripheral hepatic branches and they also hasten the polymerization of NBCA. Two $(2 / 6,33 \%)$ patients developed liver infarcts and two $(2 / 6,33 \%)$ patients underwent postembolization syndrome when coils and NBCA were used. There was only one study by Gandhi et al ${ }^{19}$ where they used coils and/or glue in 16 patients with patients with hemobilia but the etiology was different. We used only microcoils in three patients. Coils are used when the microcatheter could be positioned beyond the PSA, so that the PSA can be trapped proximally and distally with coils and no collateral vessels can fill the PSA. Alternately, coiling can be done for the entire segment. ${ }^{7,9,18}$ There have been case reports of the use of percutaneous thrombin for hepatic artery aneurysms., ${ }^{7,20,21}$ Covered stents can also be used. ${ }^{22}$

Surgery is reserved when an endovascular approach is not feasible or failed. Petrou et $\mathrm{al}^{23}$ and Mate et $\mathrm{al}^{8}$ described failure of angiographic embolization probably due to filling of pseudoaneurysm by collaterals and hence 

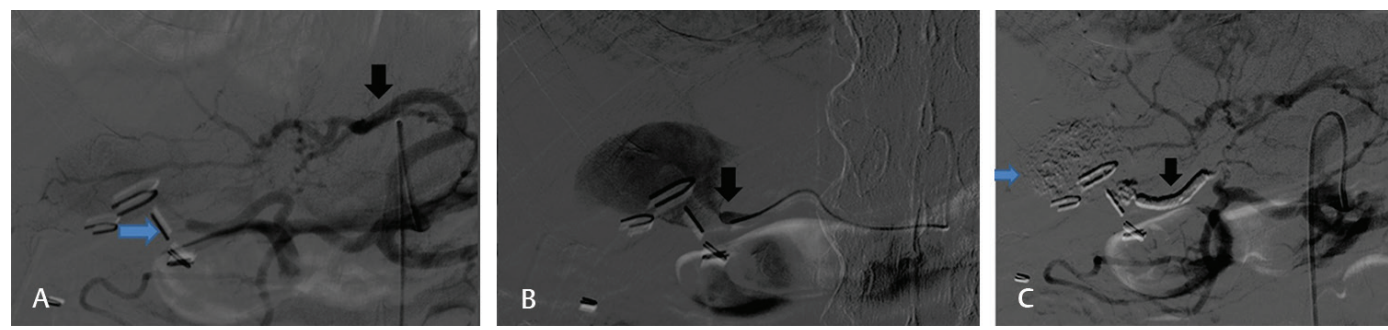

Fig. 4 (A) Celiac angiogram with 5F Simmon (Sim1) catheter showing left gastrohepatic trunk (black arrow) supplying left lobe of liver; pseudoaneurysm (blue arrow) arising from right hepatic artery. Metallic clips (black arrow) are seen near the pseudoaneurysm. (B) Superselective cannulation with microcatheter of right hepatic artery showing pseudoaneurysm and no filling of distal hepatic branches. (C) Postembolization with n-butyl cyanoacrylate (NBCA)-black arrow, celiac angiogram showing no filling of pseudoaneurysm as well as right hepatic artery. NBCA filling up pseudoaneurysm as well as right hepatic artery (RHA).

surgical management with right hepatic artery ligation was performed. Bin Traiki et $\mathrm{al}^{24}$ described stent placed in a replaced right hepatic artery PSA, which was complicated by left and proper hepatic artery PSA after 5 days and was managed by percutaneous thrombin injection. After 2 days, rebleeding occurred which was managed with gelfoam, thereby avoiding surgery. No rebleeding was seen in any of our cases.

Our study has many limitations including its retrospective nature, small sample size and lack of long-term follow-up for delayed complications.

\section{Conclusion}

In conclusion, TAE is highly effective for treating PSA following LC. NBCA or coils or both can be used as per the arterial anatomy, morphology of the artery, catheter position and operator's experience. Complications with TAE do occur and most of them can be managed conservatively.

\section{Conflicts of Interest}

None declared.

\section{References}

1 Giger U, Ouaissi M, Schmitz SF, Krähenbühl S, Krähenbühl L. Bile duct injury and use of cholangiography during laparoscopic cholecystectomy. Br J Surg 2011;98(3):391-396

2 Shamiyeh A, Wayand W. Laparoscopic cholecystectomy: early and late complications and their treatment. Langenbecks Arch Surg 2004;389(3):164-171

3 Machado NO. Biliary complications postlaparoscopic cholecystectomy: mechanism, preventive measures, and approach to management: a review. Diagn Ther Endosc 2011;2011:967017

4 Strasberg SM, Helton WS. An analytical review of vasculobiliary injury in laparoscopic and open cholecystectomy. HPB (Oxford) 2011;13(1):1-14

5 Nicholson T, Travis S, Ettles D, et al. Hepatic artery angiography and embolization for hemobilia following laparoscopic cholecystectomy. Cardiovasc Intervent Radiol 1999;22(1):20-24

6 Ahmed Madanur M, Battula N, Sethi H, Deshpande R, Heaton N, Rela M. Pseudoaneurysm following laparoscopic cholecystectomy. Hepatobiliary Pancreat Dis Int 2007;6(3): 294-298
7 Genyk YS, Keller FS, Halpern NB. Hepatic artery pseudoaneurysm and hemobilia following laser laparoscopic cholecystectomy. A case report. Surg Endosc 1994;8(3):201-204

8 Mate AD, Surnare KR, Deolekar SS, Gvalani AK. Lower gastrointestinal bleeding due to hepatic artery pseudoaneurysm following laparoscopic cholecystectomy. J Minim Access Surg 2013;9(1):31-33

9 Feng W, Yue D, ZaiMing L, ZhaoYu L, Wei L, Qiyong G. Hemobilia following laparoscopic cholecystectomy: computed tomography findings and clinical outcome of transcatheter arterial embolization. Acta Radiol 2017;58(1):46-52

10 Sansonna F, Boati S, Sguinzi R, Migliorisi C, Pugliese F, Pugliese R. Severe hemobilia from hepatic artery pseudoaneurysm. Case Rep Gastrointest Med 2011;2011:925142

11 Senthilkumar MP, Battula N, Perera M, et al. Management of a pseudo-aneurysm in the hepatic artery after a laparoscopic cholecystectomy. Ann R Coll Surg Engl 2016;98(7):456-460

12 Patel IJ, Davidson JC, Nikolic B, et al; Standards of Practice Committee, with Cardiovascular and Interventional Radiological Society of Europe(CIRSE)Endorsement. Consensus guidelines for periprocedural management of coagulation status and hemostasis risk in percutaneous image-guided interventions. J Vasc Interv Radiol 2012;23(6):727-736

13 Choi SH, Gwon DI, Ko GY, et al. Hepatic arterial injuries in 3110 patients following percutaneous transhepatic biliary drainage. Radiology 2011;261(3):969-975

14 Deziel DJ, Millikan KW, Economou SG, Doolas A, Ko ST, Airan MC. Complications of laparoscopic cholecystectomy: a national survey of 4,292 hospitals and an analysis of 77,604 cases. Am J Surg 1993;165(1):9-14

15 Roche-Nagle G, Maceneaney G, Harte P. Pseudo-aneurysm of the hepatic artery after laparoscopic cholecystectomy: a case report. J Minim Access Surg 2006;2(2):73-75

16 Quinke H. Ein Fall von Aneurysma der Leberarterie. Klin Wochenschr 1871;88:773-786

17 Machado NO, Al-Zadjali A, Kakaria AK, Younus S, Rahim MA, Al-Sukaiti R. Hepatic or cystic artery pseudoaneurysms following a laparoscopic cholecystectomy: literature review of aetiopathogenesis, presentation, diagnosis and management. Sultan Qaboos Univ Med J 2017;17(2):e135-e146

18 Chigot V, Lallier M, Alvarez F, Dubois J. Hepatic artery pseudoaneurysm following laparoscopic cholecystectomy. Pediatr Radiol 2003;33(1):24-26

19 Gandhi V, Doctor N, MararS, Nagral A, NagralS. Major hemobiliaexperience from a specialist unit in a developing country. Trop Gastroenterol 2011;32(3):214-218

20 Boddy A, Macanovic M, Thompson J, Watkinson A. Use of an endovascular stent graft and percutaneous thrombin 
injection to treat an iatrogenic hepatic artery pseudoaneurysm. Ann R Coll Surg Engl 2010 (e-pub ahead of print). doi: /147870810X12822015504806

21 Kumar A, Sheikh A, Partyka L, Contractor S. Cystic artery pseudoaneurysm presenting as a complication of laparoscopic cholecystectomy treated with percutaneous thrombin injection. Clin Imaging 2014;38(4):522-525

22 Hylton JR, Pevec WC. Successful treatment of an iatrogenic right hepatic artery pseudoaneurysm and stenosis with a stent graft. J Vasc Surg 2010;51(6):1510-1513
23 Petrou A, Brennan N, Soonawalla Z, Silva MA. Hemobilia due to cystic artery stump pseudoaneurysm following laparoscopic cholecystectomy: case presentation and literature review. Int Surg 2012;97(2):140-144

24 Bin Traiki TA, Madkhali AA, Hassanain MM. Hemobilia post laparoscopic cholecystectomy. J Surg Case Rep 2015; 2015(2):rju159-rju159 